\title{
Evaluación de fuentes de variación en pruebas multi-ambientes para rendimiento y sus componentes en algodón
}

\section{Evaluation of sources of variation in multi-environment tests for yield and its components in cotton}

Hernando Alberto Araújo-Vázquez; Miguel Mariano Espitia-Camacho²*; Milton Edinson Buelvas-Guzmán³; María Camila MuñozPuche ${ }^{4}$; Hermes Araméndiz-Tatis ${ }^{5}$

${ }^{1}$ Ing. Agrónomo, M.Sc. Corporación Colombiana de Investigación Agropecuaria -AGROSAVIA-, C.I. Turipaná. Cereté - Córdoba, Colombia; e-mail: haraujo@agrosavia.co; iDttps://orcid.org/0000-0003-3679-3973

${ }^{2}$ Ing.Agrónomo, M.Sc.. Ph.D. Universidad de Córdoba, Facultad de Ciencias Agrícolas. Montería - Córdoba, Colombia; e-mail: mmespitia@correo. unicordoba.edu.co; iD https://orcid.org/0000-0001-7382-9643

${ }^{3}$ Ing. Agrónomo, Profesional independiente. Montería - Córdoba, Colombia; e-mail: miltonbgmiltonbg@gmail.com; (iD https://orcid.org/0000-00025076-3490

${ }^{4}$ Ing. Agrónomo, Profesional independiente. Montería - Córdoba, Colombia; e-mail: camila.mupu20@gmail.com; (iD https://orcid.org/0000-00032126-4438

${ }^{5}$ Ing. Agrónomo, M.Sc.. Ph.D. Universidad de Córdoba, Facultad de Ciencias Agrícolas. Montería - Córdoba, Colombia; e-mail: haramendiz@correo. unicordoba.edu.co; iD https://orcid.org/0000-0002-2585-6273

*autor para correspondencia: mmespitia@correo.unicordoba.edu.co

Cómo citar: Araújo-Vázquez, H.A.; Espitia-Camacho, M.M.; Buelvas-Guzmán, M.E.; Muñoz-Puche, M.C.; Araméndiz-Tatis, H. 2021. Evaluación de fuentes de variación en pruebas multi-ambientes para rendimiento y sus componentes en algodón. Rev. U.D.C.A Act. \& Div. Cient. 24(2):e1849. http://doi.org/10.31910/rudca.v24.n2.2021.1849

Artículo de acceso abierto publicado por Revista U.D.C.A Actualidad \& Divulgación Cientí ica, bajo una Licencia Creative Commons CC BY-NC 4.0

Publicación o icial de la Universidad de Ciencias Aplicadas y Ambientales U.D.C.A, Institución de Educación Superior Acreditada de Alta Calidad por el Ministerio de Educación Nacional.

Recibido: enero 21 de 2021

Aceptado: julio 8 de 2021

Editado por: Ingeborg Zenner de Polanía

\section{RESUMEN}

La valoración de las fuentes de variación de los análisis de varianza combinado en las pruebas de evaluación multi-ambientes es de gran importancia en la selección de genotipos para recomendar nuevos cultivares, por su adaptabilidad y estabilidad. El objetivo de esta investigación fue evaluar la significancia estadística y contribución de las fuentes de variación: ambientes, genotipos e interacción genotipo $\mathrm{x}$ ambiente para rendimiento de algodónsemilla, porcentaje de fibra y rendimiento de fibra, en las zonas del Caribe seco y húmedo colombiano. Se utilizaron los datos de dos pruebas multi-ambientes por zona, donde se evaluaron 10 
genotipos de fibra media diferentes, bajo el diseño de bloques completos al azar, con cuatro repeticiones. Cada prueba estuvo conformada por cuatro ensayos realizados durante las cosechas 2003/2004, 2007/2008 y 2009/2010. Los resultados señalan que, en promedio, el ambiente fue la fuente de variación más importante en significancia $(\mathrm{p}<0,05$ y p <0,01) y variación $(>64 \%)$, de los análisis de varianza combinado para rendimiento de algodónsemilla y fibra, con tendencia similar en su comportamiento entre ellas y en las dos zonas del Caribe colombiano. Para el porcentaje de fibra en el Caribe seco, la fuente de variación más importante fue genotipos $(74,4 \%$ ) y en el Caribe húmedo fue el ambiente $(57,2$ $\%$ ). Se sugiere subdividir las dos zonas del Caribe en subzonas más homogéneas ambientalmente o aumentar en más de cuatro el número de ensayos por prueba, para minimizar el efecto del ambiente y la interacción genotipo $\mathrm{x}$ ambiente.

Palabras clave: Gossypium hirsutum; Rendimiento; Componentes de rendimientos; Evaluación agronómica; Análisis de varianza combinado; Caribe colombiano.

\section{ABSTRACT}

The assessment of the sources of variation of the combined analysis of variance in the multi-environment evaluation tests is of great importance in the selection of genotypes to recommend new cultivars for their adaptability and stability. The objective of this research was to evaluate the statistical significance and contribution of the sources of variation: environments, genotypes and genotype $\mathrm{x}$ environment interaction for cotton-seed yield, fiber percentage and fiber yield, in the dry and humid Colombian Caribbean zones. Data from two multi-environment tests per zone were used, where 10 different medium fiber genotypes were evaluated, under a randomized complete block design with four replications. Each test consisted of four experimentos carried out during the 2003/2004, $2007 / 2008$ and $2009 / 2010$ harvests. The results indicate that on average the environment was the most important source of variation in significance $(p<0.05$ and $p<0.01)$ and variation $(>64$ $\%$ ) of the combined analysis of variance for cotton-seed yield and fiber, with a similar trend in their behavior between them and in the two zones of the Colombian Caribbean. For the percentage of fiber in the dry Caribbean, the most important source of variation was genotypes $(74.4 \%)$ and in the humid Caribbean it was the environment $(57.2 \%)$. It is suggested to subdivide the two zones of the Caribbean into more environmentally homogeneous subzones and / or to increase the number of trials per test, to minimize the effect of the environment and the genotype $\mathrm{x}$ environment interaction.

Keywords: Gossypium birsutum; Yield; Yield components; Agronomic evaluation; Combined analysis of variance; Colombian Caribbean.

\section{INTRODUCCIÓN}

El cultivo de algodón es el más importante para la producción de fibra en todo el mundo, principalmente, la especie Gossypium birsutum L. Toda la producción de fibra es utilizada en la fabricación de tejidos, de textiles y de confecciones y de las semillas, se puede obtener aceite, la harinolina, como forraje crudo, abono o combustible (Ali et al. 2018; Xing et al. 2019).

El rendimiento y sus componentes son de gran interés económico y de naturaleza poligénica, en donde las expresiones de sus genes son afectadas por factores ambientales y la interacción de los genes con el ambiente (Kang, 2020).

La región Caribe colombiana presenta dos zonas productoras, que se denominan Caribe seco y Caribe húmedo colombiano y se diferencian por su marcado contraste edafo-climático, específicamente, por la mayor fertilidad de suelos y precipitaciones en el Caribe húmedo (Burbano-Figueroa et al. 2018; Ideam, 2020). Estas diferencias ambientales afectan el rendimiento de algodón y sus componentes, principalmente, cuando se presentan excesos o déficit de lluvias en etapas críticas del cultivo (Sierra et al. 2017; Burbano-Figueroa et al. 2018); por tal razón, en aras de ser mucho más competitivas las regiones productoras, se deben identificar las localidades más propicias para la evaluación de cultivares y ello redunde en el resurgimiento de la economía, con cultivares con mayor capacidad de amortiguación, a factores bióticos y abióticos.

La importancia de las fuentes de variación ambientes (A), genotipos (G) e interacción GxA en los análisis de varianza combinado contribuye, de manera importante, para determinar el número de ambientes óptimos en que los genotipos deben ser evaluados, con el objetivo de lograr la precisión necesaria, para medir las diferencias entre genotipos, estratificar los ambientes y zonificar los genotipos promisorios (Teodoro et al. 2019; Kang, 2020).

En el mundo y en Colombia, se han realizado estudios en el cultivo de algodón, para las variables de rendimiento y sus componentes, con el fin de determinar la significancia estadística y la contribución de las fuentes de variación al análisis de varianza combinado, con soportes de estudios de adaptabilidad y de estabilidad fenotípica, para identificar, zonificar, recomendar y liberar, comercialmente, nuevos genotipos para áreas productoras (Teodoro et al. 2019; Shahzad et al. 2019; Mejía-Salazar et al. 2020); sin embargo, en Colombia no existen resultados comparativos entre zonas productoras para determinar la contribución de las fuentes de variación al análisis de varianza combinado, para el rendimiento y sus componentes en el cultivo de algodón, en el Caribe colombiano.

El objetivo fue evaluar la significancia estadística y la contribución de las fuentes de variación del análisis de varianza combinado en las pruebas de evaluación multi-ambientes, para el rendimiento de algodón-semilla (RENDAS), porcentaje de fibra (POFIB) y rendimiento de fibra (RENDIF), en el Caribe seco y húmedo colombiano, para comparar la influencia de cada componente en el proceso de evaluación, zonificación y mejorar la eficiencia del proceso de selección de las dos zonas.

\section{MATERIALES Y MÉTODOS}

Localización. Fueron utilizados los resultados de cuatro pruebas 
de evaluación agronómicas multi-ambientes, del Caribe colombiano. Dos de las pruebas fueron realizadas en el Caribe seco y dos en el Caribe húmedo colombiano, durante las cosechas 2003/2004, 2007/2008 y 2009/2010 (Cuadro 1). Cada ensayo de evaluación fue sembrado dentro de lotes comerciales de productores en cada zona, por tanto, el manejo agronómico de los mismos fue el que el agricultor aplicó a su cultivo comercial. La investigación fue realizada en el marco de la alianza estratégica entre CORPOICA y la Universidad de Córdoba, por un tiempo determinado, lo cual, limitó el uso de otros años.

Variables independientes. Correspondieron a 10 genotipos de algodón, de fibra media, conformados por siete líneas promisorias, en las cosechas 2003/2004, del Caribe seco y Caribe húmedo colombiano y ocho líneas promisorias, en las cosechas 2007/2008, en el Caribe seco y 2009/2010, en el Caribe húmedo. Los datos de cada prueba correspondieron a un grupo de 10 genotipos diferentes y fueron suministrados por el programa de mejoramiento genético de algodón de CORPOICA, cuya investigación fue realizada con la participación de la Universidad de Córdoba (Cuadro 2).

Diseño experimental. Los datos utilizados fueron obtenidos de pruebas de evaluación agronómica multi-ambientes sembrados en campo, bajo el diseño experimental de bloques completos al azar, con 10 genotipos diferentes y cuatro repeticiones, amparados en la investigación conjunta y supervisadas por el ICA y de acuerdo con la resolución No. 00148, del 18 de enero del 2005.

Variables dependientes. Las variables evaluadas fueron las siguientes: rendimiento de algodón - semilla (RENDAS), en kg.ha ${ }^{-1}$; porcentaje de fibra (POFIB), en \%, obtenido del desmote experimental de 50 motas de algodón con semillas y rendimiento

Cuadro 1. Localidades del Caribe seco y Caribe húmedo colombiano durante las cosechas algodoneras 2003/2004, 2007/2008 y 2009/2010, donde se realizaron las pruebas multi-ambientes.

\begin{tabular}{|c|c|c|c|c|c|c|c|}
\hline No. & Localidad & Departamento & $\begin{array}{l}\text { Año de } \\
\text { cosecha }\end{array}$ & $\begin{array}{c}\mathrm{T} \\
\left({ }^{\circ} \mathrm{C}\right)\end{array}$ & $\begin{array}{c}\text { PP } \\
(\mathrm{mm})\end{array}$ & $\begin{array}{c}\text { ASNM } \\
(\mathrm{m})\end{array}$ & $\begin{array}{c}\text { Fertilidad } \\
\text { De } \\
\text { Suelos }\end{array}$ \\
\hline 1 & Riohacha (Maicao) & Guajira & \multirow{4}{*}{$\begin{array}{l}2003 / 2004 \\
\text { Caribe seco }\end{array}$} & 28,2 & 621 & 53 & Baja \\
\hline 2 & El Copey & Cesar & & 27,5 & 1369 & 80 & Baja \\
\hline 3 & Codazzi (Motilonia) & Cesar & & 28,8 & 1605 & 180 & Media \\
\hline 4 & San Juan del Cesar & Guajira & & 29,5 & 1100 & 250 & Baja \\
\hline 1 & Riohacha (Maicao) & Guajira & \multirow{4}{*}{$\begin{array}{l}2007 / 2008 \\
\text { Caribe seco }\end{array}$} & 28,2 & 621 & 53 & Baja \\
\hline 2 & Codazzi (Motilonia) & Cesar & & 28,8 & 1605 & 180 & Media \\
\hline 3 & San Juan del Cesar & Guajira & & 29,5 & 1100 & 250 & Baja \\
\hline 4 & Valledupar & Cesar & & 28,1 & 961 & 138 & Media \\
\hline 1 & Cereté (Retiro de los indios) & Córdoba & \multirow{4}{*}{$\begin{array}{c}\text { 2003/2004 } \\
\text { Caribe } \\
\text { húmedo }\end{array}$} & 28 & 1300 & 20 & Media \\
\hline 2 & San Pelayo (Corocito) & Córdoba & & 27 & 1500 & 20 & Media \\
\hline 3 & Cotorra (La Culebra) & Córdoba & & 27 & 1500 & 20 & Media \\
\hline 4 & Aguachica & Cesar & & 27,7 & 1456 & 220 & Media \\
\hline 1 & $\begin{array}{l}\text { Cereté (Retiro de los indios } \\
\text { 1) }\end{array}$ & Córdoba & \multirow{6}{*}{$\begin{array}{c}\text { 2009/2010 } \\
\text { Caribe } \\
\text { húmedo }\end{array}$} & 28 & 1300 & 20 & Media \\
\hline 2 & Cereté (Manguelito 1) & Córdoba & & 27 & 1220 & 12 & Media \\
\hline 3 & $\begin{array}{c}\text { Km } 3 \text { vía Cereté - Ciénaga } \\
\text { de Oro }\end{array}$ & Córdoba & & 27 & 1220 & 12 & Media \\
\hline 4 & Ciénaga de Oro - Malagana & Córdoba & & 27 & 1220 & 33 & Media \\
\hline 5 & $\begin{array}{c}\text { Cereté (Retiro de los indios } \\
\text { 2) }\end{array}$ & Córdoba & & 28 & 1300 & 20 & Media \\
\hline 6 & Cerete (Manguelito 2) & Córdoba & & 27 & 1220 & 12 & Media \\
\hline
\end{tabular}

$\mathrm{T}=$ temperatura media en grados centígrados; $\mathrm{PP}=$ precipitación promedia anual en milímetros; ASNM = altura sobre el nivel del mar en metros; La cosecha 2009/2010 es la única que presenta 6 localidades (ambientes); Cada ambiente tiene 10 genotipos sembrados con 4 repeticiones. 
Cuadro 2. Genotipos evaluados en el Caribe seco y Caribe húmedo colombiano durante las cosechas algodoneras 2003/2004, 2007/2008 y 2009/2010.

\begin{tabular}{|c|c|c|c|}
\hline $\mathbf{N}^{\circ}$ & $\begin{array}{l}\text { 2003/2004 } \\
\text { Caribe seco }\end{array}$ & $\begin{array}{c}2007 / 2008 \\
\text { Caribe seco }\end{array}$ & Características \\
\hline 1 & LC-149 L & -Cer 0014 & Línea promisoria de fibra media \\
\hline 2 & LC-151 L & -Cer 0016 & Línea promisoria de fibra media \\
\hline 3 & LC-153 L & -Cer 0034 & Línea promisoria de fibra media \\
\hline 4 & LT-1 L & -Cer 0044 & Línea promisoria de fibra media \\
\hline 5 & LT-2 S & EL-ORO BLANCO & Línea promisoria de fibra media \\
\hline 6 & LT-3 L & -Cer 0060 & Línea promisoria de fibra media \\
\hline 7 & LT-4 L & -Cer 0007 & Línea promisoria de fibra media \\
\hline 8 & CM-123 L & -Cer 0046 & Línea promisoria de fibra media \\
\hline 9 & SM-137 S & INUANA M - $137 \mathrm{~V}$ & ariedad Colombiana de fibra media (Testigo) \\
\hline $10 \mathrm{D}$ & P-opal N & uopal & Variedad Americana de fibra media (Testigo) \\
\hline $\mathbf{N}^{\circ}$ & $\begin{array}{c}2003 / 2004 \\
\text { Caribe húmedo }\end{array}$ & $\begin{array}{c}2009 / 2010 \\
\text { Caribe húmedo }\end{array}$ & Características \\
\hline 1 & LC-149 L & C.159 & Línea promisoria de fibra media \\
\hline 2 & LC-151 L & C. 160 & Línea promisoria de fibra media \\
\hline 3 & LC-153 L & C164 L & ínea promisoria de fibra media \\
\hline 4 & LT-1 L & C.165 & Línea promisoria de fibra media \\
\hline 5 & LT-2 L & C.166 & Línea promisoria de fibra media \\
\hline 6 & LT-3 L & C.168 & Línea promisoria de fibra media \\
\hline 7 & LT-4 L & C.169 & Línea promisoria de fibra media \\
\hline 8 & CM-123 L & C.158 & Línea promisoria de fibra media \\
\hline 9 & SM-137 O & RO B.M $151 \mathrm{~V}$ & ariedad Colombiana de fibra media (Testigo) \\
\hline $10 \mathrm{D}$ & P-opal D & ELTAOPAL & Variedad Americana de fibra media (Testigo) \\
\hline
\end{tabular}

de fibra (RENDIF), en kg.ha ${ }^{-1}$, obtenido mediante la siguiente ecuación: [(RENDAS x POFIB) / 100], para cada unidad experimental y genotipo.

Análisis estadísticos de los datos. Con los datos obtenidos en las variables evaluadas, se realizó el análisis de varianza combinado, con el programa computacional de libre acceso GENES, versión Windows GENES V.2016.6.0 (Cruz, 2016), asumiendo un modelo mixto, con genotipos, como efectos fijos y ambientes, como efectos aleatorios. La significancia estadística de cada fuente de variación se realizó mediante la prueba de $\mathrm{F}$, a través del uso de los cuadrados medios y la contribución de las fuentes de variación, se hizo ponderando en porcentaje la variación de ambientes- $A$, genotipos-G e interacción genotipo por ambiente-GxA, sobre el total de las sumas de cuadrados de estas tres fuentes de variaciónFV (Meredith et al. 2012). La media para las tres variables por zona se obtuvo promediando los resultados de cada variable de las dos pruebas consideradas por zona. Los objetivos específicos planteados en este estudio no demandaron pruebas de medias, análisis de adaptabilidad y de estabilidad fenotípica, para los diferentes genotipos en cada prueba por zona.

\section{RESULTADOS Y DISCUSIÓN}

La calidad de los experimentos está asociada con la precisión de esta medida, a través del coeficiente de variación, por lo que, a menor coeficiente, mayor es la confiabilidad de los datos. En ese orden de ideas, las variables estudiadas registraron coeficientes 
acordes a los reportados por otros investigadores en esta especie, siendo siempre mayor los asociados al rendimiento de algodón semilla y rendimiento de fibra (Komala et al. 2018; Mejía-Salazar et al. 2020), con relación a porcentaje de fibra, por la complejidad genética de su herencia y los efectos no genéticos bióticos, como plagas y abióticos, como precipitación, número de horas luz, sequía y sus interacciones sobre dichos genes, que influyen en el valor fenotípico (Ribeiro et al. 2018; Campbell et al. 2018; Kang, 2020).

Rendimiento de algodón - semilla (RENDAS). Los resultados del RENDAS, se presentan en la tabla 1. La media para la zona Caribe seco fue menor en, aproximadamente, $709 \mathrm{~kg}^{\mathrm{ha}}{ }^{-1}$, con respecto al Caribe húmedo, debido a las mejores condiciones edafo-climáticas que ofrecen las sub-zonas productoras del Caribe húmedo, como suelos, precipitación y manejo agronómico, para que los cultivares expresaran su potencial genético del rendimiento (Burbano-Figueroa et al. 2018; Ideam, 2020).

Por otra parte, se encontró que los efectos ambientales fueron más importantes, tanto en el Caribe seco como en el Caribe húmedo en ambas cosechas, en comparación con la interacción genotipo por ambiente y la fuente de variación de genotipos (Tabla 1). Estos resultados son coherentes con los reportados por Campbell et al. (2011), Gul et al. (2016) y Ribeiro et al. (2018), quienes indicaron la existencia de variabilidad en la oferta ambiental entre dichas zonas agrícolas y, consecuentemente, el desempeño de los genotipos, lo cual, justifica un estudio detallado del comportamiento de cada cultivar, con el fin de identificar la magnitud de sus interacciones con el medio ambiente y reconocer aquellos con mayor estabilidad agronómica.

Esta variabilidad ambiental, se explica por factores no genéticos impredecibles, como precipitación, número de horas luz, brillo solar y manejo agronómico, que influyen en la expresión del potencial genético y su capacidad de amortiguación a los efectos ambientales, como lo sostienen Cotrim et al. (2019), Bakhsh et al. (2019) y Mejía-Salazar et al. (2020), en razón a que el rendimiento de algodón semilla, por ser un carácter cuantitativo, es afectado por el ambiente.

La fuente de variación GxA mostró un aporte intermedio a la variación en las dos cosechas del Caribe seco y Caribe húmedo (Tabla 1). Solo presentó diferencias significativas $(\mathrm{p}<0,01)$ para la cosecha 2009/2010 del Caribe húmedo, a diferencia del Caribe seco,

Tabla 1. Análisis de varianzas combinados para rendimiento algodón - semilla (RENDAS), porcentaje de fibra (POFIB) y rendimiento de fibra (RENDIF), en el Caribe seco (CS) y húmedo (CH) colombiano.

\begin{tabular}{|c|c|c|c|c|c|c|c|c|c|c|c|}
\hline \multirow[t]{2}{*}{ FV } & \multicolumn{2}{|c|}{$\mathrm{CH}$} & \multicolumn{2}{|c|}{ CS } & \multicolumn{2}{|c|}{$\begin{array}{l}\text { CH34- } \\
\text { CS34 }\end{array}$} & \multicolumn{2}{|c|}{$\mathbf{C H}$} & \multicolumn{2}{|c|}{ CS } & $\begin{array}{c}\text { CH910- } \\
\text { CS78 }\end{array}$ \\
\hline & 2003 & 2004 & 2003 & 2004 & Difer & ncia & 2009 & & 2007 & 2008 & Diferencia \\
\hline \multicolumn{12}{|c|}{ Comparación del RENDAS entre Caribe húmedo - Caribe seco en \%SC } \\
\hline G & \multicolumn{2}{|c|}{18,5} & \multicolumn{2}{|c|}{1,5} & \multicolumn{2}{|c|}{17,0} & \multicolumn{2}{|c|}{3,8} & \multicolumn{2}{|c|}{3,6} & 0,2 \\
\hline A & \multicolumn{2}{|c|}{59,9} & \multicolumn{2}{|c|}{92,6} & \multicolumn{2}{|c|}{$-32,7$} & \multicolumn{2}{|c|}{83,7} & \multicolumn{2}{|c|}{87,5} & $-3,8$ \\
\hline GxA & \multicolumn{2}{|c|}{21,6} & \multicolumn{2}{|c|}{5,9} & \multicolumn{2}{|c|}{15,7} & \multicolumn{2}{|c|}{12,6} & \multicolumn{2}{|c|}{8,9} & 3,7 \\
\hline \multicolumn{12}{|c|}{ Comparación del POFIB entre Caribe húmedo - Caribe seco en \%SC } \\
\hline G & \multicolumn{2}{|c|}{49,2} & \multicolumn{2}{|c|}{79,4} & \multicolumn{2}{|c|}{$-30,2$} & \multicolumn{2}{|c|}{20,3} & \multicolumn{2}{|c|}{69,5} & $-49,2$ \\
\hline A & \multicolumn{2}{|c|}{44,1} & \multicolumn{2}{|c|}{17,8} & \multicolumn{2}{|c|}{26,3} & \multicolumn{2}{|c|}{70,3} & \multicolumn{2}{|c|}{18,1} & 52,2 \\
\hline GxA & & & & & & & 9 & & & & $-3,0$ \\
\hline & & npar & ión de & REN1 & IF ent & Cari & húmed & - Car & e seco & $n \% S$ & \\
\hline G & & & & & & & 1, & & & & $-3,7$ \\
\hline A & & & & & & & 81 & & & & $-2,7$ \\
\hline GxA & & & & & & & 16 & & & & 6,4 \\
\hline & & Ipara & ón pro & nedia & ibe 1 & imed & $\mathrm{CH})-$ & ibe & $\cos (\mathrm{CS}$ & en $\% s$ & \\
\hline 70 & & & $\overline{\mathrm{END} A}$ & & & POF & & & ENDI & & Efecto del \\
\hline $2 \mathrm{U}$ & & $G$ & $\mathrm{~A}$ & GxA & $G$ & A & GxA & G & $\mathrm{A}$ & GxA & Ambiente \\
\hline Media & $\mathrm{CH}$ & 11,2 & 71,8 & 17,1 & 34,8 & 57,2 & 8,1 & 18,2 & 64,0 & 17,9 & Importante \\
\hline Media & el CS & 2,6 & 90,1 & 7,4 & 74,4 & 18,0 & 7,6 & 4,7 & 87,2 & 8,1 & $\begin{array}{c}\text { Muy } \\
\text { Importante }\end{array}$ \\
\hline Difer & cias & 8,6 & $-18,3$ & 9,7 & $-39,7$ & 39,3 & 0,4 & 13,4 & $-23,2$ & 9,8 & $\begin{array}{c}\text { Mayor en } \\
\text { CS }\end{array}$ \\
\hline
\end{tabular}

$\mathrm{FV}=$ fuentes de variación; $\% \mathrm{SC}=$ suma de cuadrados en porcentaje $(\%) ; \mathrm{CM}=$ cuadrados medios; $\mathrm{G}=$ genotipo; $\mathrm{A}=$ ambiente; $\mathrm{GxA}=$ interacción; * = significativo al $5 \%(\mathrm{p}<0,05) ; * *=$ significativo al $1 \%(\mathrm{p}<0,01)$; ns = no significativo; Media de RENDAS y RENDIF = en kilogramos por hectárea (kg.ha-1); Media de POFIB = en porcentaje (\%); CV = coeficiente de variación en porcentaje $(\%)$; Diferencia = resta entre $\mathrm{CH}-\mathrm{CS}$; Media del $\mathrm{CH}=$ promedio de las cosechas del $\mathrm{CH}(\mathrm{CH} 34 / \mathrm{CH} 910)$; Media del $\mathrm{CS}=$ promedio de las cosechas del CS (CS34/CS78). 
donde ambas cosechas no presentaron diferencias significativas y esto es, posiblemente, a que los genotipos presentaron una mayor capacidad de amortiguación individual, a las oscilaciones ambientales (Tabla 1). Resultados similares han sido reportados previamente por Campbell et al. (2011), Mukoyi et al. (2018) y Cotrim et al. (2019), donde la interacción no superó los valores del $25 \%$, en la contribución de la suma de cuadrados y es la que menos aporta a la variación y tienen gran validez, dadas las diferencias agroecológicas, entre dichas zonas productoras.

En lo concerniente con la fuente de variación genotipos (G), además de contribuir en menor grado a explicar, tanto la variación en las sumas de cuadrados en el Caribe seco como en el Caribe húmedo, no registró diferencias significativas en tres de las cuatro cosechas, lo cual, refleja igual comportamiento agronómico y las diferencias con relación a otras cosechas, se sustentan en los efectos ambientales, por lo que la regionalización de las áreas de producción es necesaria, para que se exprese el mayor potencial genético de los genotipos (Meredith et al. 2012; Mukoyi et al. 2018; Cotrim et al. 2019; Bakhsh et al. 2019; Mejía-Salazar et al. 2020), a través de cultivares con adaptación específica y se logren mejores producciones de algodón semilla, aprovechando la oferta ambiental.

Porcentaje de fibra (POFIB). Los resultados de ambas zonas algodoneras (Tabla 1), señalan que la media para el Caribe húmedo fue menor en, aproximadamente, $3 \%$, con respecto al Caribe seco y se debe al menor peso de las semillas, dada la existencia de una correlación genética alta e inversa entre el porcentaje de fibra y el peso de semilla, como lo reportan Xing et al. (2019) y Amein et al. (2020).

La suma de cuadrados para genotipos explicó, en mayor proporción, la variación total en las dos cosechas del Caribe seco y Caribe húmedo. En el Caribe seco hubo mayor contribución, con valores superiores en 2003/2004 y 2007/2008, en comparación con el Caribe húmedo, donde se presentaron participación de genotipos menores a $50 \%$, en la cosecha 2003/2004 y menores del $21 \%$, en la cosecha 2009/2010.

Los resultados encontrados en el presente estudio obedecen a la divergencia genética de los genotipos y la correlación entre fenotipo y genotipo, por el menor número de genes involucrados en su control genético, a pesar de ser un carácter poligénico afectado por el ambiente, por lo que interfiere en el valor fenotípico (Xing et al. 2019), lo cual, coincide con lo reportado por Ribeiro et al. (2018) y Albuquerque et al. (2020), quienes afirman que POFIB varía, principalmente, por los efectos del genotipo y lo reportan con una participación en las sumas de cuadrados, superiores al $57 \%$.

En ambas zonas y entre cosechas, los cuadrados medios presentaron diferencias significativas $(\mathrm{p}<0,01)$ para ambientes; ello se explica por ambientes contrastantes y los efectos del trópico, dado que factores abióticos, como las precipitaciones, la temperatura, la humedad relativa y la radiación y los factores bióticos, como plagas y enfermedades, influenciados por las condiciones de cada sub-zona o cosecha, especialmente, en el Caribe seco, por tener menor régimen de precipitación, hay menor aprovechamientos de nutrientes y formación de asimilados con destino a las semillas, siendo estas menos pesadas y, por ende, con mayor porcentaje de fibra. Estos resultados concuerdan con los encontrados por Campbell et al. (2011), Farias et al. (2016), Shahzad et al. (2019) y Mejía-Salazar et al. (2020), quienes reportan que la fuente de variación ambientes fue significativa $(\mathrm{p}<0,01$ y $\mathrm{p}<0,05)$, para POFIB.

La contribución en las sumas de cuadrados por la interacción GxA fue menor, para explicar la variación fenotípica de los datos en las dos cosechas en ambas zonas. Sus valores fueron menores a 9,5\%, en el Caribe húmedo, para las cosechas 2003/2004 y 2009/2010, con significancia estadística, lo que indica que los cultivares modificaron su clasificación en función de la oferta ambiental, mientras que en el Caribe seco, fue alrededor del $3 \%$, para 2003/2004 y cercano al $12 \%$, para $2007 / 2008$ y no significativos, posiblemente, por tener buena capacidad de amortiguación individual de los genotipos a las oscilaciones ambientales que, al provenir de cruzamientos diferentes, poseen cierta variabilidad genética y su clasificación no cambia en las diferentes condiciones ambientales del Caribe seco, lo cual, es opuesto en el Caribe húmedo; ello permitiría seleccionar genotipos superiores, como lo señalan Campbell et al. (2011; 2012), Meredith et al. (2012), Ribeiro et al. (2018), Shahzad et al. (2019), Mejía-Salazar et al. (2020) y Albuquerque et al. (2020), sobre todo, cuando la interacción GxA no supera el $20 \%$ de la contribución en las sumas de cuadrados y es la que menos contribuye.

Rendimiento de fibra (RENDIF). Con diferencias de 137 $\mathrm{kg} \cdot \mathrm{ha}^{-1}$, los resultados obtenidos para RENDIF fueron superiores en el Caribe húmedo con respecto al Caribe seco (Tabla 1). La mayor contribución a la suma de cuadrados fue dada por el ambiente en las dos zonas de estudio, seguido de la interacción genotipo por ambiente, excepto para el Caribe húmedo, en la temporada 2003/2004 y por último el genotipo, coherentes con los reportados por Teodoro et al. (2019), Shahzad et al. (2019) y Mejía-Salazar et al. (2020); esto significa que, factores ambientales no controlables, como número de horas luz, brillo solar, precipitación, algunas propiedades del suelo, jugaron un rol muy importante en la expresión del potencial genético y la existencia de correlación genética, entre RENDAS y RENDIF (Mukoyi et al. 2018; Teodoro et al. 2019; Amein et al. 2020) y su influencia en la interacción genotipo ambiente, que resultó en un $75 \%$ de los períodos de estudio, no significativa y de poca magnitudes, como lo señalan Shahzad et al. (2019) y Mejía-Salazar et al. (2020), quienes sostienen que la interacción GxA, para RENDIF, en las sumas de cuadrados es explicada con valores menores al $15 \%$; por tal razón, el número de experimentos para identificar los cultivares con mayor estabilidad agronómica, debe estar soportado en una mayor cantidad de experimentos, para estar mucho más seguro en el proceso de selección y de liberación de variedades, como lo sostienen Nadeem et al. (2018).

Genotipos fue la fuente de variación menos importante en el Caribe seco, explicando menos del 5,3 \% de la variación de la suma de cuadrados en las dos cosechas 2003/2004 y 2007/2008, mientras 
que fue intermedia en el Caribe húmedo, contribuyendo con alrededor del $35 \%$, en el $2003 / 2004$ y siendo la menos importante en la cosecha 2009/2010, con menos del $2 \%$ de la variación. La fuente genotipos fue significativa $(\mathrm{p}<0,05$ y $\mathrm{p}<0,01)$ en la cosecha $2003 / 2004$, para ambas zonas, lo que indica una mayor expresión del potencial y divergencia genética, con mayor contribución en el Caribe húmedo, lo cual, se refleja en la media del RENDIF, con $1.570 \mathrm{~kg} \cdot \mathrm{ha}^{-1}$, aproximadamente.

Comparación de las variables dependientes y zonas. Los resultados de las tres variables dependientes RENDAS, POFIB y RENDIF, para las dos zonas, se presentan en la tabla 2. En la comparación de medias, se observa que para RENDAS y RENDIF, la mayor contribución a las sumas de cuadrados fue la fuente de variación ambientes, que presentó mayor efecto en ambas cosechas del Caribe seco y Caribe húmedo, lo que corrobora los ambientes de esta zona del país con contrastantes; asimismo, el ambiente tiene un efecto colateral adicional sobre la interacción GxA, la cual, cuando resulta significativa, como en el Caribe húmedo, genera cambios en la clasificación de los genotipos entre los ambientes, enmascara la expresión del carácter de interés, disminuye la correlación entre los valores fenotípicos y genotípicos, reduce el progreso de la selección y dificulta la labor de selección y posterior recomendación de los genotipos, para una o varias localidades (Shahzad et al. 2019; Kang,
2020). La suma de cuadrado para genotipos en estas dos variables registró poca variación y significancia para RENDAS en el Caribe húmedo, período 2003/2004 y Caribe seco y Caribe húmedo para RENDIF, en la temporada 2003/2004, señalando diferencias genéticas en algunas oportunidades y, en otras, no se manifiestan por el enmascaramiento que ejerce el ambiente, dificultando el proceso de selección (Shahzad et al. 2019; Kang, 2020).

La variable POFIB, se comportó de manera diferente, debido a que la fuente genotipos realizó la mayor contribución a las sumas de cuadrados en ambas cosechas del Caribe seco y Caribe húmedo, excepto para la cosecha 2009/2010, indicando que los genotipos responden de acuerdo con su genética y condiciones de manejo agronómico, razón por la cual, la ausencia de lluvias, al final del ciclo, favorece semillas más pequeñas y menor peso con mayor porcentaje de fibra. Lo antes anotado, se sustenta en la participación de la suma de cuadrados de ambientes, como la segunda en importancia y la reducida participación, a dicha suma de cuadrados, de la interacción genotipo por ambiente.

Para minimizar los efectos del ambiente, se han sugerido dos caminos: a) dividir las zonas productoras de evaluación en subzonas más homogéneas, con menor variabilidad ambiental dentro de cada subzona y mayor variabilidad entre las subzonas, lo cual, permite

Tabla 2. Comparación de los análisis de varianzas combinados del rendimiento algodón - semilla (RENDAS), porcentaje de fibra (POFIB) y rendimiento de fibra (RENDIF), en el Caribe seco (CS) y húmedo $(\mathrm{CH})$ colombiano y la comparación entre zonas productoras.

\begin{tabular}{|c|c|c|c|c|c|c|c|c|c|c|c|}
\hline \multirow[t]{2}{*}{ FV } & \multicolumn{2}{|c|}{$\mathrm{CH}$} & \multicolumn{2}{|c|}{ CS } & \multicolumn{2}{|c|}{$\begin{array}{c}\text { CH34- } \\
\text { CS34 }\end{array}$} & \multicolumn{2}{|c|}{$\mathrm{CH}$} & \multicolumn{2}{|c|}{ CS } & $\begin{array}{c}\text { CH910- } \\
\text { CS78 }\end{array}$ \\
\hline & \multicolumn{2}{|c|}{$2003 / 2004$} & \multicolumn{2}{|c|}{$2003 / 2004$} & \multicolumn{2}{|c|}{ Diferencia } & \multicolumn{2}{|c|}{$2009 / 2010$} & \multicolumn{2}{|c|}{$2007 / 2008$} & Diferencia \\
\hline \multicolumn{12}{|c|}{ Comparación del RENDAS entre Caribe húmedo - Caribe seco en \%SC } \\
\hline G & \multicolumn{2}{|c|}{18,5} & \multicolumn{2}{|c|}{1,5} & \multicolumn{2}{|c|}{17,0} & \multicolumn{2}{|c|}{3,8} & \multicolumn{2}{|c|}{3,6} & 0,2 \\
\hline A & \multicolumn{2}{|c|}{59,9} & \multicolumn{2}{|c|}{92,6} & \multicolumn{2}{|c|}{$-32,7$} & \multicolumn{2}{|c|}{83,7} & \multicolumn{2}{|c|}{87,5} & $-3,8$ \\
\hline GxA & \multicolumn{2}{|c|}{21,6} & \multicolumn{2}{|c|}{5,9} & \multicolumn{2}{|c|}{15,7} & \multicolumn{2}{|c|}{12,6} & \multicolumn{2}{|c|}{8,9} & 3,7 \\
\hline \multicolumn{12}{|c|}{ Comparación del POFIB entre Caribe húmedo - Caribe seco en \%SC } \\
\hline G & \multicolumn{2}{|c|}{49,2} & \multicolumn{2}{|c|}{79,4} & \multicolumn{2}{|c|}{$-30,2$} & \multicolumn{2}{|c|}{20,3} & \multicolumn{2}{|c|}{69,5} & $-49,2$ \\
\hline A & & & & & & & 70 & & 18 & & 52,2 \\
\hline GxA & & & & & & & 9 & & 12 & & $-3,0$ \\
\hline & & $\mathrm{np}$ & ín de & REN & ent & $\mathrm{C}$ & aúmec & $-\mathrm{Ca}$ & e seco & en $\% \mathrm{SC}$ & \\
\hline G & & & & & & & & & 5 & & $-3,7$ \\
\hline A & & & & & & & 8 & & 8 & & $-2,7$ \\
\hline GxA & & & & & & & & & & & 6,4 \\
\hline & & ipara & ón pre & nedia & aribe 1 & imed & CH) - & aribe & $\operatorname{co}(\mathrm{CS})$ & en $\% \mathrm{~S}$ & \\
\hline 70 & & & END & & & $\mathrm{POF}$ & & & ENDI & & Efecto del \\
\hline & & G & A & GxA & G & A & GxA & G & A & GxA & Ambiente \\
\hline Media & $\mathrm{elCH}$ & 11,2 & 71,8 & 17,1 & 34,8 & 57,2 & 8,1 & 18,2 & 64,0 & 17,9 & Importante \\
\hline Media & el CS & 2,6 & 90,1 & 7,4 & 74,4 & 18,0 & 7,6 & 4,7 & 87,2 & 8,1 & $\begin{array}{c}\text { Muy } \\
\text { Importante }\end{array}$ \\
\hline Difer & cias & 8,6 & $-18,3$ & 9,7 & $-39,7$ & 39,3 & 0,4 & 13,4 & $-23,2$ & 9,8 & $\begin{array}{l}\text { Mayor en } \\
\text { CS }\end{array}$ \\
\hline
\end{tabular}

$\mathrm{FV}=$ fuentes de variación; $\% \mathrm{SC}=$ suma de cuadrados en porcentaje $(\%) ; \mathrm{CM}=$ cuadrados medios; $\mathrm{G}=$ genotipo; $\mathrm{A}=$ ambiente; $\mathrm{GxA}$ $=$ interacción; $*=$ significativo al $5 \%(\mathrm{p}<0,05) ; * *=$ significativo al $1 \%(\mathrm{p}<0,01)$; ns = no significativo; Diferencia $=$ resta entre CHCS; Media del $\mathrm{CH}=$ promedio de las cosechas del CH (CH34/CH910); Media del CS = promedio de las cosechas del CS (CS34/CS78). 
reducir o minimizar el efecto de ambientes y de interacción GxA y b) aumentar el número de ensayos por prueba de evaluación por zona en más de cuatro, para reducir los efectos de ambientes y GxA y se permita, en ambos casos, identificar, seleccionar y recomendar los genotipos superiores en cada sub-zona, con base en su valor genotípico real (Cotrim et al. 2019; Kang, 2020). Frente a las posiciones anteriores surge una pregunta adicional: ¿Esto es funcional y viable en los países, como Colombia, ubicados en la zona tropical o subtropical, que se caracterizan, precisamente, por una alta variabilidad edafo-climática impredecible?

Los resultados obtenidos permiten concluir que, en promedio, el ambiente fue la fuente más importante con significancia estadística, para explicar la variación en los análisis de varianza combinado, para rendimiento de algodón-semilla y fibra, con tendencia similar en su comportamiento entre ellas y en las dos zonas del Caribe colombiano. Para el porcentaje de fibra en el Caribe seco, la fuente de variación más importante fue genotipos y en el Caribe húmedo fue el ambiente. Se sugiere subdividir las dos zonas del Caribe en subzonas, más homogéneas ambientalmente o aumentar, en más de cuatro, el número de ensayos por prueba, para minimizar el efecto del ambiente y la interacción genotipo $\mathrm{x}$ ambiente.

Conflictos de intereses: El manuscrito fue preparado y revisado con la participación de todos los autores, quienes declaramos que no existe ningún conflicto de intereses que ponga en riesgo la validez de los resultados presentados. Financiación: Este estudio fue cofinanciado por la Universidad de Córdoba y los autores.

\section{REFERENCIAS}

1. ALBUQUERQUE, R.R.S.D.; CAVALCANTI, J.J.V.; FARIAS, F.J.C.; QUEIROZ, D.R.; CARVALHO, L.P.D. 2020. Estimates of genetic parameters for selection of colored cotton fiber. Rev. Caatinga. (Brasil). 33(1):253-259.

http://dx.doi.org/10.1590/1983-21252020v33n127rc

2. ALI, I.; KHAN, N.U.; RAHMAN, M.; GUL, R.; BIBI, Z.; GUL, S.; AHMED, S.; ALI, S.; ALI, N.; AFRIDI, K.; HAQ, H.A. 2018. Genotype by environment and biplot analyses for yield and fiber traits in upland cotton. Int. J. Agric. Biol. (Pakistán). 20(1):1979-1990.

https://doi.org/10.17957/IJAB/15.0720

3. AMEIN, M.M.M.; MASRI, M.I.; EL-ADLY, H.H.; ATTTIA, S.S. 2020. Correlation and path coefficient analysis for yield components traits in egyptian cotton genotypes. (Gossypium barbadense L.). Plant Arch. (Egipto). 20(2):803-806.

4. BAKHSH, A.; REHMAN, M.; SALMAN, S.; ULLAH, R. 2019. Evaluation of cotton genotypes for seed cotton yield and fiber quality traits under water stress and non-stress conditions. Sarhad J. Agric. (Pakistán). 35(1):161-170.

http://dx.doi.org/10.17582/journal.sja/2019/35.1.161.170

5. BURBANO-FIGUEROA, O.; MONTES-MERCADO, K.S.;
PASTRANA-VARGAS, I.J.; CADENA-TORRES, J. 2018. Introducción y desarrollo de variedades de algodón Upland en el sistema productivo colombiano: Una revisión. Cien. Agri. (Colombia). 15(1):29-44.

https://doi.org/10.19053/01228420.v15.n1.2018.7754

6. CAMPBELL, B.T.; CHEE, P.W.; LUBBERS, E.; BOWMAN, D.T.; MEREDITH, W.R.; JOHNSON, J.; FRASER, D.E. 2011. Genetic improvement of the Pee Dee cotton germplasm collection following seventy years of plant breeding. Crop sci. (USA). 51(3):955-968.

https://doi.org/10.2135/cropsci2010.09.0545

7. CAMPBELL, B.T.; CHEE, P.W.; LUBBERS, E.; BOWMAN, D.T.; MEREDITH, W.R.; JOHNSON, J.; JONES, D.C. 2012. Dissecting genotype $x$ environment interactions and trait correlations present in the Pee Dee cotton germplasm collection following seventy years of plant breeding. Crop sci. (USA). 52(2):690-699. https://doi.org/10.2135/cropsci2011.07.0380

8. CAMPBELL, B.T.; DEVER, J.K.; HUGIE, K.L.; KELLY, C.M. 2018. Cotton fiber improvement through breeding and biotechnology. In: Cotton Fiber: Physics, Chemistry and Biology. Springer, Cham. (USA). p.193-215. https://doi.org/10.1007/978-3-030-00871-0_10

9. COTRIM, M.F.; FARIAS, F.J.C.; DE CARVALHO, L.P.; TEODORO, L.P.R.; BHERING, L.L.; TEODORO, P.E. 2019. Environmental stratification in the Brazilian cerrado on the yield and fiber quality of cotton genotypes. Bioscience J. (Brasil). 35(5):1349-1355. https://doi.org/10.14393/BJ-v35n5a2019-42259

10. CRUZ, C.D. 2016. Programa Genes V.2016.6.0 - Aplicativo computacional em genética e estatística. Disponible desde internet en:

http://www.ufv.br/dbg/genes/genes.htm (con acceso 22/09/2016).

11. FARIAS, F.J.C.; DE CARVALHO, L.P.; DA SILVA FILHO, J.L.; TEODORO, P.E. 2016. Correlations and path analysis among agronomic and technological traits of upland cotton. Genet. Mol. Res. (Inglaterra). 15(3):1-7. http://dx.doi.org/10.4238/gmr.15038239

12. GUL, S.; KHAN, N.U.; GUL, R.; BALOCH, M.; LATIF, A.; KHAN, I.A. 2016. Genotype by environment and phenotypic adaptability studies for yield and fiber variables in upland cotton. J. Anim. Plant Sci. (Pakistán). 26(3):776-786.

13. INSTITUTO DE HIDROLOGÍA, METEOROLOGÍA Y ESTUDIOS AMBIENTALES, IDEAM. 2020. Tiempo y clima. Datos solicitados. Disponible desde internet en: http://www.ideam.gov.co/web/tiempo-y-clima (con acceso 23/08/2020). 
14. KANG, M.S. 2020. Genotype-environment interaction and stability analyses: an update. Quantitative genetics, genomics and plant breeding (USA). (Ed. 2). p.140-161. https://doi.org/10.1079/9781789240214.0140

15. KOMALA, M.; MEENAKSHI, N.; KUMAR, M. 2018. Genetic variability, Heritability and Correlation Analysis in F2 Populations of Ratoon Upland Cotton Hybrids. IJAEB (India). 11(6):815-827.

16. MEJÍA-SALAZAR, J.R.; GALEANO-MENDOZA, C.H.; BURBANO-HERAZO, E.; VALLEJO-CABRERA, F.A.; ARANGO, M. 2020. Interacción genotipo por ambiente de nueve variedades de algodón para los Valles interandinos en Colombia. Agron. Mesoam (Costa Rica). 31(1):31-42. https://doi.org/10.15517/AM.V31I1.37178

17. MEREDITH JR., W.R.; BOYKIN, D.L.; BOURLAND, F.M.; CALDWELL, W.D.; CAMPBELL, B.T.; GANNAWAY, J.R.; GLASS, K.; PHILLIPS JONES, A.; MAY, L.M.; SMITH, C.W.; ZHANG, J. 2012. Genotype x environment interactions over seven years for yield, yield components, fiber quality, and gossypol traits in the regional high-quality tests. J. Cotton Sci. (USA). 16(1):160-169.

18. MUKOYI, F.; GASURA, E.; MAKUNDE, G.S. 2018. Implications of correlations and genotype by environment interactions among cotton traits. Afr. Crop Sci. J. (Uganda). 26(2):219-235.

https://doi.org/10.4314/acsj.v26i2.5

19. NADEEM, F.; KHAN, N.; KHALID, S.; AZAM, S.; SAEED, B.; JAN, T.; KHAM, K.; KHAM, M. 2018. Genotype $\times$ environment interaction studies in F5 populations of upland cotton under agro-climatic condition of Peshawar. Pure Appl. Biol. (Pakistan). 7(3):973-991. http:/ /dx.doi.org/10.19045/bspab.2018.700116

20. RIBEIRO, L.P.; CARVALHO L., P.D.; FARIAS F., J.C.; RODRIGUES J., I.D.S.; TEODORO, P.E.; BHERING, L.L. 2018. Genetic gains in agronomic and technological traits of elite cotton genotypes. Bragantia. (Brasil). 77(3):466-475. https://doi.org/10.1590/1678-4499.2017329

21. SHAHZAD, K.; QI, T.; GUO, L.; TANG, H.; ZHANG, X.; WANG, H.; QIAO, X.; ZHANG, M.; ZHANG, B.; FENG, J.; SHAHID, M.; WU, J.; XING, C. 2019. Adaptability and stability comparisons of inbred and hybrid cotton in yield and fiber quality traits. Agron. (USA). 9(9):516-528.

https://doi.org/10.3390/agronomy9090516

22. SIERRA, M.; ESPITIA, M.; CADENA, J. 2017. Correlación entre rendimiento, estabilidad fenotípica y métodos de selección simultánea en algodón. Rev. Temas Agrarios. (Colombia). 22(1):21-30.

https://doi.org/10.21897/rta.v22i1.912

23. TEODORO, P.E.; FARIAS F., J.C.; DE CARVALHO, L.P.; RIBEIRO, L.P.; NASCIMENTO, M.; AZEVEDO, C.F.; CRUZ, C.D.; BHERING, L.L. 2019. Adaptability and stability of cotton genotypes regarding fiber yield and quality traits. Crop Sc. (USA). 59(2):518-524.

https://doi.org/10.2135/cropsci2018.04.0250

24. XING, H.; YUAN, Y.; ZHANG, H.; WANG, L.; MAO, L.; TAO, J.; WANG, X.; FENG, W.; WHANG, H.; WHANG, Q.; WEI, Z.; ZHANG, G.; LIU, X.; LI, Z.; LIANG, X.; ZHEN, X. 2019. Multi-environments and multi-models association mapping identified candidate genes of lint percentage and seed index in Gossypium birsutum L. Mol Breeding (China). 39(1):149-165.

https://doi.org/10.1007/s11032-019-1063-7 